General Conference (Part B)

\title{
LOW-COMPLEXITY MULTIUSER DETECTOR FOR COHERENT AND INCOHERENT CDMA MOBILE RADIO COMMUNICATIONS
}

\author{
E. Del Re, R. Fantacci, S. Morosi, M. Raugei, G.Vivaldi. \\ LENST Lab. \\ E. E. Dept. Of University of Florence, \\ Via di S. Marta 3, \\ I-50139 Florence, Italy
}

\begin{abstract}
This paper deals with a multiuser detector based on a decorrelating approach for asynchronous up-link DS/CDMA communications in which QPSK modulation schemes are used in transmission and coherent and incoherent receivers in reception. It is shown here that the receivers proposed are near-far resistant and exhibits lower complexity than optimum detector. The incoherent realization allows: a lighter complexity overhead for the synchronization circuitry. Receivers performance is expressed in terms of bit error rate (BER) which has been derived by simulations under the assumption of a slowly frequency-selective Rayleigh fading channel.
\end{abstract}

\section{INTRODUCTION}

Code Division Multiple Access (CDMA) systems have been strongly considered as key techniques for the future wireless communications.

As it is known, CDMA systems main shortcoming is their interference limited nature: in mobile radio communications classical Rake receiver has been demonstrated to be impaired by the so-called near-far effect due to Multiple Access Interference (MAI). Researchers efforts [1-3] have been concentrated on designing multiuser detectors (MUDs) capable of reducing these negative phenomena.

Among different linear multiuser detection techniques, decorrelating approach [4] is based on the idea of performing inverse operation of crosscorrelation matrix, so recovering, for each user, original informative signals as they'd flow out of the communication channel, with the only interference due to additive White Gaussian Noise (AWGN). Moreover, decorrelating detector is optimal according to maximum-likelihood criterion when received amplitudes are unknown.

The resistance of these receiver to the near-far problem implies promising outlook for this detection technique in personal communications. The main drawback here is the implementation complexity which has so far prevented its complete development. The implementation complexity of a multiuser detection scheme may be reduced by the sliding window decorrelating algorithm (SLWA) proposed by Wijayasuria et al. [5]. This is a practical and flexible approach suitable for applications where the number of users is high and changing with time. A modified version of the Wijayasuria et al.'s approach was recently proposed by Yoon et al. [6] and by Zheng et al. [7]. In this case, the cross-correlation from the past and future symbols in the asynchronous channels is eliminated (see Fig. 1) by using the frame format and periodical insertion of a zero-energy bit in transmission.

This paper deals with an extension of the Yoon et al.'s method [6] to the case of a QPSK modulation scheme and slowly frequency selective Rayleigh communication channel both for coherent and incoherent receivers. In particular, the QPSK scheme has been considered in order to allow a better bandwidth occupancy with respect to the BPSK scheme. Differently from [6], it is necessary to account for interference effects due to both phase and quadrature components for either coherent or incoherent detection. This result cannot be obtained as in the BPSK modulated signals case [8], so that a suitable phase compensation algorithm, outlined in section II, is needed here in order to perform a coherent detection [9]. Incoherent reception has to be accomplished accordingly to proper differential procedure, addressed in the same section too. It is worth stressing that incoherent receiver gives up carrier phase recovery with lighter complexity load. Section III deals with detector performance in comparisons with the classical Rake receiver in terms of BER. Finally concluding remarks are given in section IV.

\section{IL. SYSTEM MODEL}

A communication system with $K$ simultaneous users is considered herein. Each user transmits a $N$ symbols sequence, according to the QPSK modulation scheme. Alternatively $2 N$ bits are supposed to be transmitted on phase and quadrature components. Let $n(t)$ be the AWGN introduced by the communication channel: under the assumption of using the same spreading sequence for the phase and quadrature components, we have [1]: 


$$
\begin{aligned}
& \left.\underline{\underline{y}}_{F}(l)+\underline{\mathrm{j}}_{\underline{\rho}}(l)=\underline{\underline{R}}(-1) \underline{\underline{C}}(l+1) \underline{\underline{f}}(l+1)+\mathrm{j} \underline{q}(l+1) \mid+\underline{\underline{R}}(0) \underline{\underline{C}} \underline{\underline{C}}(l) \underline{\underline{L}}(l)+\mathrm{j} \underline{\underline{g}}(l)\right\rfloor
\end{aligned}
$$

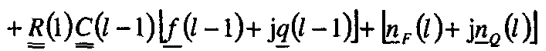

where $\underline{f}(l)+\underline{\mathrm{j}} \underline{(l)}$ is the complex vector composed of the transmitted data, i.e., $\underline{f}(l)=\left[f_{1}(l), f_{2}(l), \ldots, f_{K L}(l)\right]$, where $f_{k l}(l) \in\{ \pm 1\}$. Each vector is comprised of $K L$ elements, where $L$ is the number of replicas of each user signal which can be discriminated at the receiving end. Each replica is considered as an individual user, so that it is still possible to exploit multiuser detection and to take advantage of the Rake diversity technique. Then, $\underline{y}_{F}(l)+\underline{\mathrm{j}}_{Q}(l)$ is the complex vector composed of the matched filter outputs, $\underline{\underline{C}}(l)$ are the diagonal matrices whose components are complex channel fading gains of average energy $E_{b}$, i.e., $c_{k}(l)=\sqrt{w_{k}(l)} e^{j v_{k}(l)}$, where $w_{k}(l)$ is the received power from the $l^{\text {th }}$ bit of the $k^{\text {th }}$ user and $\vartheta_{k}(l)$ the random phase introduced by the multipath fading channel; $\underline{=}(l)$ are the $K L \times K L$ normalized signal crosscorrelation matrices defined in [4] and $\underline{n}_{F}(l)+\underline{j}_{Q}(l)$ is due to AWGN with zero mean and one-sided power spectral density equal to $N_{0}$.

Since $C(l)$ are complex matrices, it is possible to assume $\underline{\underline{C}}(l)=\underline{\underline{C}}_{F}(l)+\underline{\underline{j}}_{Q}(l)$. Thus, complex vectorial equation (1) can be decomposed in two real vectorial equations, namely:

$$
\begin{aligned}
& \left.\underline{y}_{F}(l)=\underline{\underline{R}}(-1) \underline{\underline{C}} \underline{\underline{C}}_{F}(l+1) \underline{f}(l+1)-\underline{\underline{C}}(l+1) \underline{q}(l+1)\right]+\underline{\underline{R}}(0) \underline{\underline{C}} \underline{\underline{C}}_{F}(l) \underline{f}(l)-
\end{aligned}
$$

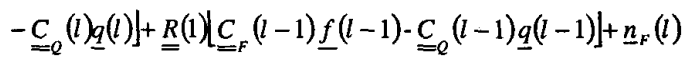

$$
\left.\underline{y}_{Q}(l)=\underline{\underline{R}}(-1) \underline{\underline{C}}_{F}(l+1) \underline{q}(l+1)+\underline{\underline{C}}_{Q}(l+1) \underline{f}(l+1)\right]+\underline{\underline{R}}(0) .(3)
$$

$$
\left.\left.\cdot \underline{\underline{C}}_{F}(l) \underline{q}(l)+\underline{\underline{C}}_{Q}(l) \underline{f}(l)\right]+\underline{\underline{R}}(1) \underline{\underline{C}} \underline{\underline{C}}_{F}(l-1) \underline{q}(l-1)+\underline{\underline{C}}_{Q}(l-1) \underline{f}(l-1)\right]+\underline{\underline{n}}_{Q}(l)
$$

where transmitted vectors $\underline{f}(l)$ and $\underline{q}(l)$ have to be somehow determined. Towards this end, we define: $\underline{F}(l)=\underline{W}_{F}(l) \underline{f}(l)-\underline{\underline{W}}_{Q}(l) \underline{q}(l)$

$$
\underline{Q}(l)=\underline{\underline{W}}_{F}(l) \underline{q}(l)+\underline{\underline{W}}_{Q}(l) \underline{f}(l) \quad \forall l=0, \ldots . N-1
$$

Hereinafter, we focus only on equation: we will extend later the results obtained to the second equation. We considered a sequence formed by $P$ bits (window) for each user. By means of some algebraic manipulations, we have:

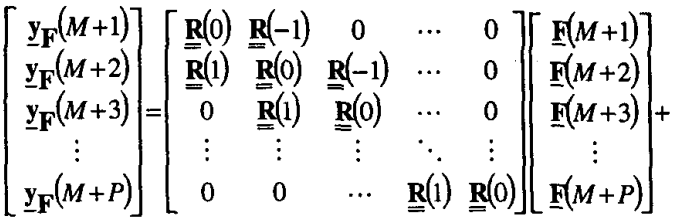

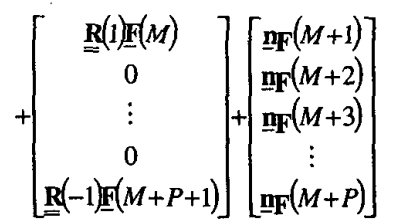

where matrix $R$, defined as

$$
\mathcal{R}=\left[\begin{array}{ccccc}
\mathbf{R}(0) & \mathbf{R}(-1) & 0 & \ldots & 0 \\
\overline{\underline{\mathbf{R}}(1)} & \overline{\mathbf{R}}(0) & \underline{\mathbf{R}(-1)} & \ldots & 0 \\
0 & \overline{\overline{\mathbf{R}}}(1) & \underline{\mathbf{R}}(0) & \ldots & 0 \\
\vdots & \vdots & \underline{\vdots} & \ddots & \vdots \\
0 & 0 & \cdots & \underline{\underline{\mathbf{R}}(1)} & \underline{\underline{\mathbf{R}}}(0)
\end{array}\right]
$$

is the crosscorrelation Toeplitz matrix, $M$ is an offset from the start of the transmission and $P<N$ is the data temporal window length within the complete received sequence. The linear system (5) is composed of $P$ equations with $P+2$ unknown values; in particular, $\underline{y}_{F}(M+1)$ is dependent on $\underline{F}(M)$ and, likewise, $\underline{y}_{F}(M+P)$ on $\underline{F}(M+P+1)$. Thus, we need to know these values to solve the linear system (6). One possible solution to this problem is to assume $M=0$ and $P=N$ (in this way $F(M)$ and $F(M+P+1)$ are both equal to zero), but this is equivalent to considering the entire received sequence. This particular case is considered in [4] where matrix $R$ is a $N K L \times N K L$ matrix. This makes the inversion of $R$ not feasible for practical values of the sequence length $N$. In order to avoid this drawback, we have adopted here a suitable approach. Our strategy is to assume terms $\underline{F}(M)$ and $\underline{F}(M+P+1)$ in (5) both equal to zero. This condition is fulfilled by means of the periodical insertion of a zero-energy bit in the informative bit sequence. So, the interference due to the cross-correlation between the first and last symbols of each window with the past and future symbols, respectively, can be eliminated (see Fig. 1), in any asynchronous environment. Then, parameter $P$ has been chosen in order to obtain a feasible solution of the linear system (5).

From Fig. 1.a it can be seen that the transmission is arranged so that the relative differences in arrival times of 
the zero-energy bits at the base-station are smaller than the single-bit duration $T_{b}$, i.e., $\left|\tau_{k}-\tau_{l}\right| \leq T_{b}, \quad \forall k, l$.

We note that the overhead needed to fulfil the synchronization constraints is not too heavy to be unacceptable [8]. Now, the linear system (5) can be solved by exploiting the block tridiagonal structure of crosscorrelation matrix $R$ thanks to the Sliding Window algorithm [7]. As described in [1], the decorrelation process results in a noise variance enhancement, but it does not affect its spectrum properties.

Thus, all the values of vectors $\underline{F}(l)$ are determined. Then, $Q(l)$ is obtained by solving a similar linear system. This means that two decorrelation blocks must be used. After performing these iterations, the mutual interference among users has been eliminated, but random phase offset introduced by the multipath fading channel is still to be faced.

\section{II.a Coherent Scheme}

In the coherent scheme considered here perfect knowledge of $\vartheta_{k}(l)$ is assumed, while $w_{k}(l)$ has an unknown value. After decorrelating operation a phase compensation block has been introduced. In particular, the $k^{\text {th }}$ element of (4) is equal to:

$\left\{\begin{array}{l}F_{k}(l)=\sqrt{w_{k}(l)} f_{k}(l) \cos \vartheta_{k}(l)-\sqrt{w_{k}(l)} q_{k}(l) \sin \vartheta_{k}(l) \quad \forall l=1, \ldots, \mathrm{N}-1(6) \\ Q_{k}(l)=\sqrt{w_{k}(l)} q_{k}(l) \cos \vartheta_{k}(l)+\sqrt{w_{k}(l)} f_{k}(l) \sin \vartheta_{k}(l)\end{array}\right.$

As can be easily seen, (6) is a simple linear system composed of two equations with two unknown values, $\sqrt{w_{k}(l)} f_{k}(l)$ and $\sqrt{w_{k}(l)} q_{k}(l)$. Its solution is given by the following operations:

$\left\{\begin{array}{l}\sqrt{w_{k}(l)} f_{k}(l)=F_{k}(l) \cos \vartheta_{k}(l)+Q_{k}(l) \sin \vartheta_{k}(l) \quad \forall l=1, \ldots, \mathrm{N}-1 \\ \sqrt{w_{k}(l)} q_{k}(l)=Q_{k}(l) \cos \vartheta_{k}(l)-F_{k}(l) \sin \vartheta_{k}(l)\end{array}\right.$

In this way all the values of vectors $f(l)$ are determined. Then, $q(l)$ is obtained by solving a similar linear system.

We emphasize that the simplicity of this solution is due to our choice of using only one pseudo-noisy sequence for each QPSK user.

Finally, the values of the decision variables are obtained via Equal Gain Combining (EGC) of each user different replicas.

Replicas values are affected only by the AWGN and by the attenuation given by the multipath fading, but they are completely free from the interference caused by the other users and the random phase deviation introduced by the multipath fading channel.

\section{II.b Incoherent Scheme}

If incoherent approach is adopted, transmitted bits effective value is determined by differential detection upon decorrelation block outputs, by taking advantage of a proper window structure with some light differences with the frame considered in Fig.1. In particular, in the incoherent scheme window structure considered here (Fig. 3.a), unitary value bits are supposed to be first transmitted, after zero-energy ones, either for the phase than for the quadrature component.

These reference bits make possible differential detection of the successive values inside the frame, through utilization of the circuit described in Fig. 2: in particular, classical differential procedure involve multiplication of decorrelator blocks actual outputs with complex conjugated of previous one and this operation is executed for all the bits following the reference: in the considered slowly fading environment, this multiplication is nearly equivalent to MRC, since replicas complex attenuation $c_{k}(l)$ is supposed not to change significantly from a bit to the successive.

For what concerns bits values analytical determination, if $\operatorname{diag}[F(l)]$ and diag $[Q(l)]$ are supposed to be the diagonal matrices whose non zero elements are equal to the elements of the vectors $\underline{F}(l)$ and $\underline{Q}(l)$ and incoherent demodulators outputs $\underline{D}_{F}(l)$ and $\underline{D}_{Q}(l)$, are subject to the reference constraint upon the first bits of the frame, i.e.,

$$
\underline{D}_{F}(1)=1, \underline{D}_{q}(1)=1
$$

successive bits values are obtained as in the following

$$
\begin{aligned}
& \left.\underline{D}_{F}(l)=\{\underline{\operatorname{diag}}[F(l)] \underline{F}(l-1)+\underline{\underline{\operatorname{diag}}} \underline{\underline{Q}}(l)] \underline{Q}(l-1)\right\} \underline{D}_{F}(l-1)- \\
& -\{\operatorname{diag} \underline{Q}(l)\rfloor \underline{F}(l-1)-\operatorname{diag}[F(l)] \underline{Q}(l-1)\}_{D_{Q}}(l-1)
\end{aligned}
$$

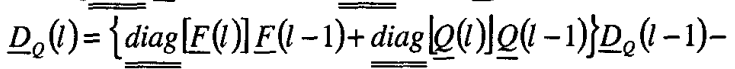

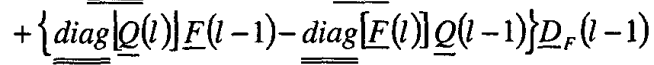

$$
\begin{aligned}
& \forall l=2, \ldots, P
\end{aligned}
$$

Decision variables determination is now straightforward: different replicas contributions are summed for each user and for each component. As in the coherent scheme, these values are affected only by AWGN and attenuation caused by multipath fading, but free from the multiple access interference. Phase deviation due to multipath fading phenomena are suppressed unless extremely fast variations are considered: in fact, differentially .coded bits has been transmitted with initialization given by the reference bits whose insertion has to be made once per window in order to adapt this detection scheme to sliding window algorithm non continuos structure. 
General Conference (Part B)

\section{SIMULATION RESULTS}

In performing our simulations the following conditions have been assumed:

- Symbol rate for both modulations equal to 31.496 Ksymbol/sec;

- One Gold sequence (with processing gain equal to 127) assigned to each QPSK or DQPSK user;

-Ten interfering users communication system.

We consider an up-link Rayleigh multipath fading suburban channel: in the model considered, whose description can be found in [10], six replicas can be resolved distinguished at the receiver input.

Figs. 3-4 show the BER performance of proposed coherent multiuser detector as a function of the ratio between the energy per bit at the receiving end $\left(E_{b}\right)$ and $N_{0}$, for different values of Signal to Interference Ratio (SIR). BER performance obtained by classical Rake receiver with the same number of interfering users and with a single user is also shown in the figures for comparison purposes. The good behavior of the multiuser detector is apparent in Figs. 3-4, where BER performance of the proposed multiuser detector is independent of the near-far effect.

The results shown in Figs. 3-4 have been obtained by inserting a zero-energy symbol every four information bits $(P=4)$. This leads to an efficiency equal to $80 \%$. This value is clearly too low for practical applications. The dependence of the BER performance of the proposed receiver on the frame length is investigated in Fig. 5 for the case of four interfering users and three resolvable paths. The power unbalance between the desired user signal and the interfering signals has been set to $10 \mathrm{~dB}$. This figure proves that the BER performance is not dependent on the frame length; hence, high efficiencies are guaranteed.

For what concerns differential detection schemes, same working conditions and simulation sets have been assumed. In the first simulation scenario, outlined in Fig. $1 \mathrm{~b}$, transmitted informative basic frame is composed of five bits, among which the first is zero-energy and the second is devoted to reference values, so that system efficiency is equal to $60 \%$.

Fig. 6 shows incoherent basic detector performance in comparison with conventional Rake receiver results in the same environment and single user bound while Fig. 7 shows the BER performance of the same detector for different values of SIR: proposed detector performance is almost near-far independent.

Because of very low efficiency, two advanced incoherent implementations are proposed in the following:

- phase reference bits are inserted more rarely (Fig. 1.c);

- window length is increased (Fig. 1.d);

The first solution can be applied to basic incoherent scheme by means of some enhancement: i) reference bits insertion timing can be made programmable and system performance can be studied as a function of the insertion rate; moreover the insertion rate could be tied to channel variation rate, so that an adaptive solution is feasible; new reference bits insertion rate has to be known by the receiver;

ii) differential detection of the frames without reference bits has to be initialized accordingly to (10) through previous frame last bits values that are to be stored in dedicated registers.

For what concerns second solution to achieve better system efficiency, its derivation from (6) and (10) is straightforward, but, since double length windows are considered, sliding window algorithm complexity increase is remarkable.

In the first advanced scheme reference bits have been inserted once per five or ten windows, as shown in Fig. 1,c, obtaining respectively system efficiency equal to 76 and $78 \%$, but with growing performance loss in comparison with basic scheme results, reported in Fig. 8 .

In the second advanced scheme ten bit epochs length windows are considered, (Fig. 1.d), so that system efficiency is equal to $80 \%$. Performance comparison between this receiver and the basic schemes is reported in Fig. 9. Light performance loss is particularly evident for high $E_{b}$ to $N_{0}$ ratios; this phenomenon is a direct consequence of differential detection: in fact, in these receivers, errors bursts are the most likely. The longer informative frame is, the higher the probability of appearance of long errors burst. Hence, in this last scheme, interleaving techniques bring greater benefits.

\section{CONCLUDING REMARKS}

In this paper different solutions of multiuser receivers for QPSK modulated signals in DS/CDMA up-link communications has been presented for coherent and incoherent detection approaches. For incoherent realization optimum performance-complexity trade-off is determined. The near-far resistance of proposed receivers and their good behavior with respect to the classical Rake receiver have been verified by means of computer simulations in the case of a slowly frequency-selective Rayleigh fading channel.

\section{REFERENCES}

[1] Verdù S.: "Multiuser detection.", 1998, Cambridge University Press, Cambridge UK. 
[2] Duel-Hallen. A., Holtzman J., Zvonar Z.: "Multiuser Detection for CDMA Systems.", IEEE Pers. Comm., Vol.2, Apr. 1995.

[3] Moshavi S.: "Multiuser Detection for DS-CDMA communications", IEEE Comm. Mag., Vol. 34, Oct. 1996.

[4] Lupas R. and Verdù S.: "Near-far resistance of multiuser detectors in asynchronous channels.", IEEE Trans. On Commun., Vol. COM-38, April 1990.

[5] Wijayasuria, S.S.H., Norton G.H., McGeehan J.P.: "Sliding window decorrelating algorithm for DS-CDMA receivers.", Electronics Letters, Vol. 28, No. 17, August 1992.

[6] Yoon S.Y.., Hong S.E., Ahn J., Lee H.S.: "Pilot symbol aided coherent decorrelating detector for up-link CDMA

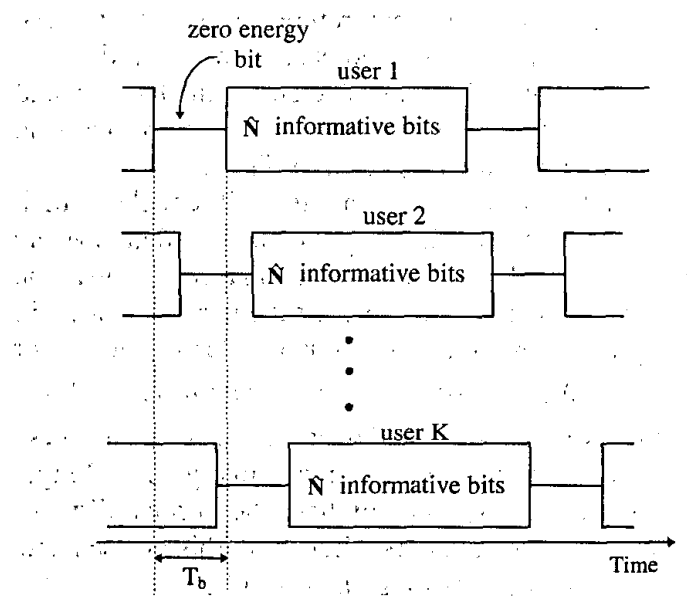

Fig. 1.a: Transmitted data frame structures (QPSK scheme).

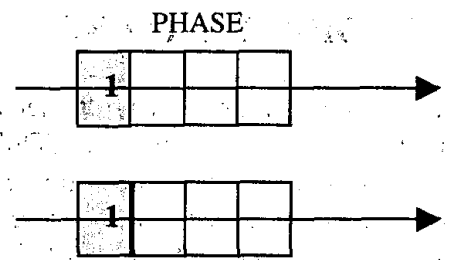

QUADRATURE

Fig. 1.b: Transmitted data frame structures (DQPSK scheme).

$$
\because \quad
$$$$
\text { MTHATHWTH }
$$
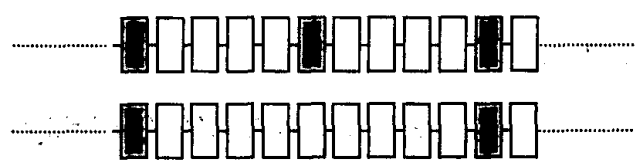

Fig. 1.c: Transmitted data frame structures (DQPSK scheme). mobile radio communication.", Electronics Letters, Vol. 30, No. 12, June 1994.

[7] Zheng F. and Barton S.: "Near-Far Resistant Detection of CDMA Signals via Isolation Bit Insertion.", IEEE Trans on Commun., Vol. COM-43, Feb. 1995.

[8] Zvonar Z., Brady D.: "Suboptimal Multiuser Detection For Frequency-Selective Rayleigh Fading Synchronous Chanels.", IEEE Trans. On Commun., Vol COM-43, Feb./Mar./Apr. 1995.

[9] Del Re E., Fantacci R., Morosi S., Vivaldi G., "A LowComplexity Multiuser Detector for Up-Link CDMA QPSK Mobile Radio Communications.", to be published on ACM Wireless Networks (1999).

[10] GSM Recommendation 05.05 (Ver. 3.7.0)

PHASE

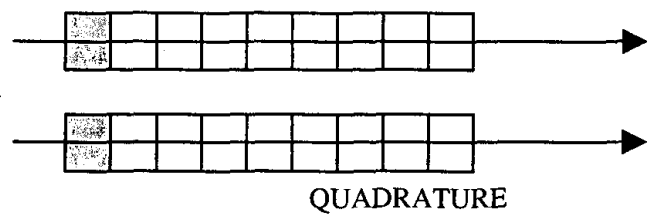

Fig. 1.d: Transmitted data frame structures (DQPSK scheme).

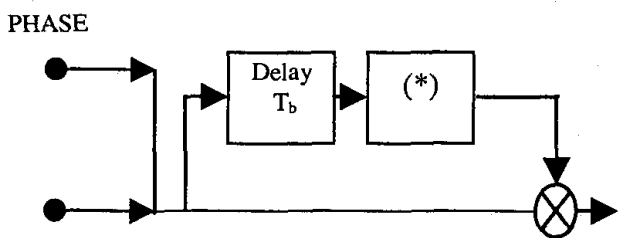

QUADRATURE

Fig. 2: Differential Decoder (DQPSK scheme).

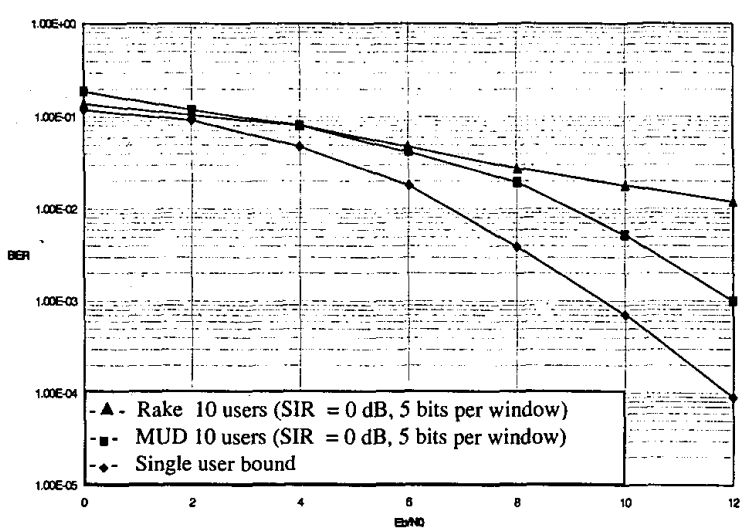

Fig. 3: Bit Error Rate comparisons (QPSK scheme, ideal power control). 


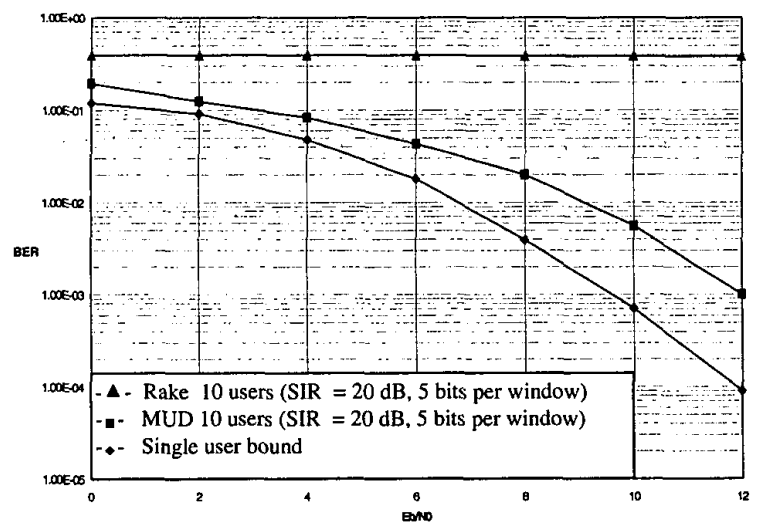

Fig. 4: Bit Error Rate comparisons (QPSK scheme, power unbalance between the desired user signal and the interfering signals equal to $20 \mathrm{~dB}$ ).

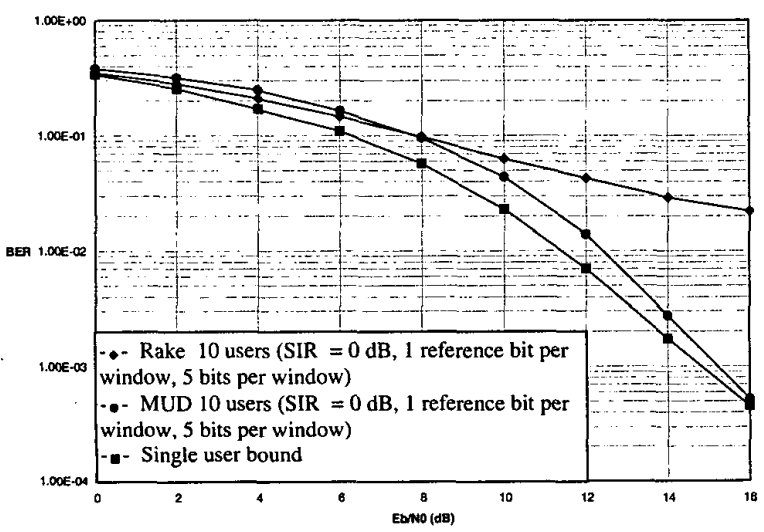

Fig. 6: Bit Error Rate comparisons (DQPSK scheme, ideal power control, 1 reference bit per window, 5 bits window)

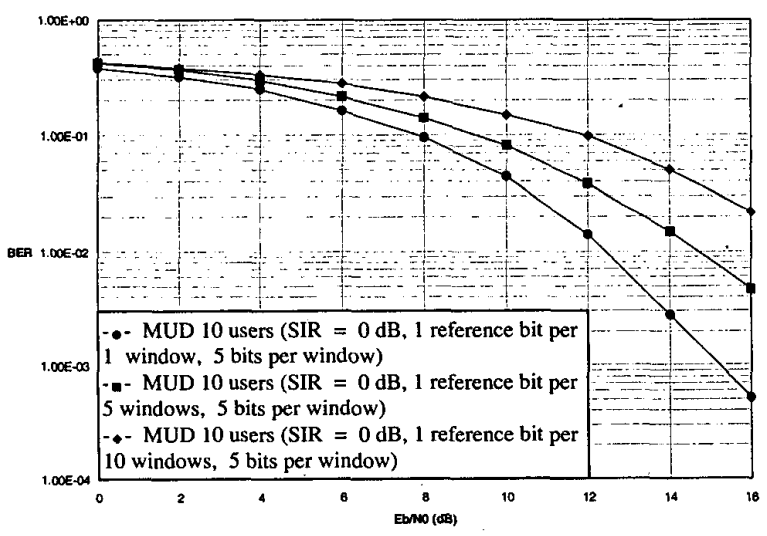

Fig. 8: Bit Error Rate comparisons (DQPSK scheme, ideal power control, 1 reference bit per 1,5 or 10 windows, 5 bits window).

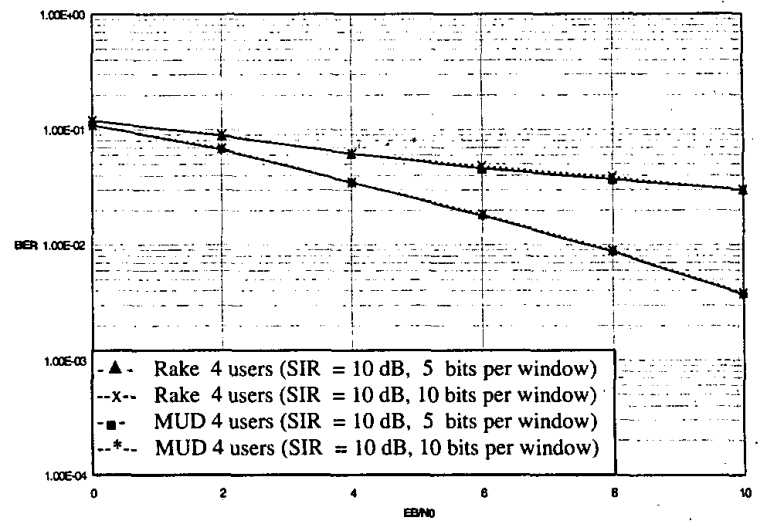

Fig. 5: Bit Error Rate comparisons (QPSK scheme, power unbalance between the desired user signal and the interfering signals equal to $10 \mathrm{~dB}$, with different frame lengths).

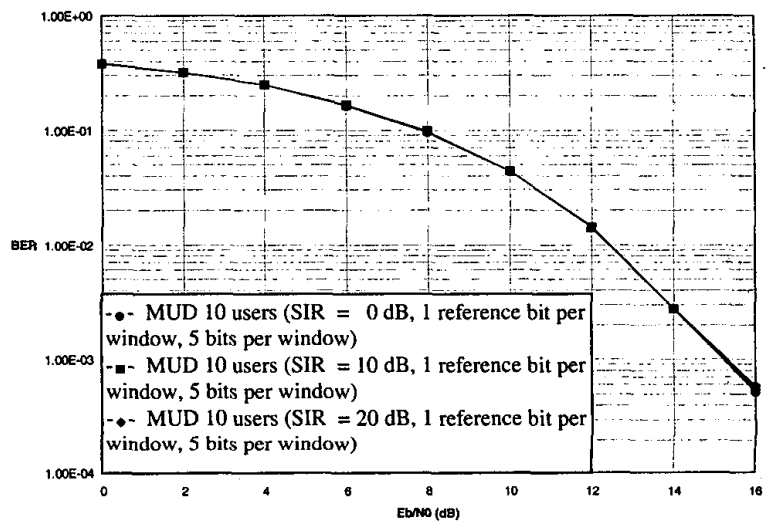

Fig. 7: Bit Error Rate comparisons (DQPSK scheme with different power unbalance between the desired user signal and the interfering signals, 1 reference bit per window, 5 bits

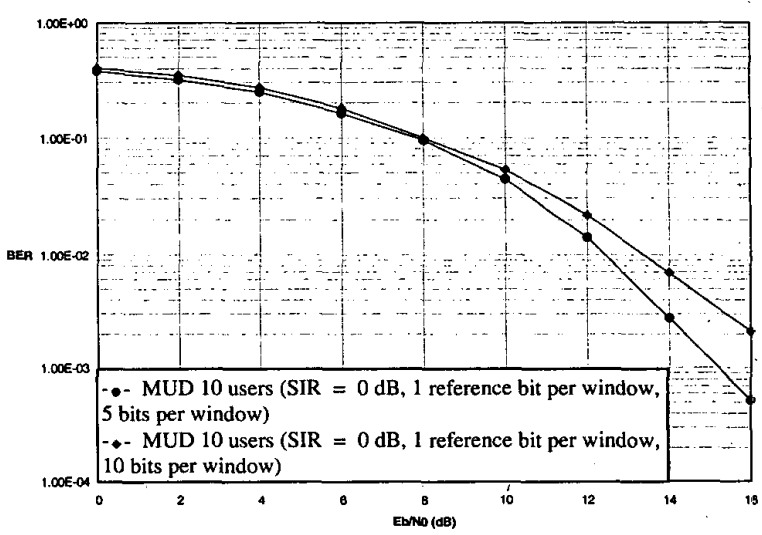

Fig. 9: Bit Error Rate comparisons (DQPSK scheme, ideal power control, 1 reference bit per window, 5 or 10 bits window). 